\title{
Performance of waterborne acrylic surface coatings on wood impregnated with Cu-ethanolamine preservatives
}

\author{
M HUMAR, M PAVLIČ, D ŽLINDRA ${ }^{\dagger}$, M TOMAŽIČ $\check{\check{C}}^{\dagger \dagger}$ and M PETRIČ* \\ Department of Wood Science and Technology, University of Ljubljana, Jamnikarjeva 101, SI 1000 Ljubljana, Slovenia \\ †Slovenian Forestry Institute, Večna pot 2, SI 1000 Ljubljana, Slovenia \\ ${ }^{\dagger}$ TIL - Institute for Wood Technology of Ljubljana, Dolenjska cesta 42, SI 1000 Ljubljana, Slovenia
}

MS received 26 July 2007; revised 7 January 2011

\begin{abstract}
Two waterborne acrylic coatings were applied on spruce wood, impregnated with two copperethanolamine containing preservatives $(\mathrm{CuE}$ and $\mathrm{CuEQ}$ ), one of them $(\mathrm{CuEQ})$ containing a boron compound, octanoic acid and a quaternary ammonium compound as additives. Lower contact angles and deeper penetration of both coating types were observed on $\mathrm{CuEQ}$ treated, compared to untreated or CuE-treated wood. Improved adhesion of coatings on CuEQ impregnated wood was exhibited as well. In general, characteristics of surface finishes on copper-ethanolamine treated wood were comparable to untreated wood. It was also shown that copper leaching from the preserved wood was significantly reduced by application of the surface finishes.
\end{abstract}

Keywords. Copper-ethanolamine wood preservatives; leaching; Norway spruce wood; waterborne acrylic surface coatings.

\section{Introduction}

Effectiveness of copper compounds against wood decay fungi makes them important constituent of several classical, novel and proposed wood preservatives. Among them, copper-amine based preservatives are currently the most attractive ones, due to foreseen limitations of arsenic and/or chromium in CCA (a wood preservative based on copper, chromium and arsenic compounds) and/or CCB (a wood preservative containing copper, chromium and boron compounds). Ethanolamine is reported as the most suitable amine source by several researchers, and it is used in a number of emerging preservative systems, including alkaline copper quats (ACQ), copper dimethyl-dithiocarbamate (CDDC), $\mathrm{Cu}-\mathrm{HDO}$ and copper azole (CA) (Cao and Kamdem 2004).

However, leaching of copper from wood, impregnated with various copper-ethanolamine based preservatives, is still higher in comparison to leaching from wood, treated with copper-chromium based ones (Ung and Cooper 2005). Copper fixation in wood, treated with copper-ethanolamine based preservatives, can be significantly improved by addition of carboxylic acids to preservative solutions. Particularly octanoic acid was found very effective, due to its hydrophobic effect and less soluble complexes formed

\footnotetext{
*Author for correspondence (marko.petric@bf.uni-lj.si)
}

between copper-ethanolamine and octanoic acid (Humar et al 2005). Furthermore, leaching of copper from impregnated wood could be additionally decreased by application of surface finishes on preserved wood. Other co-biocides, like boron compounds, azoles or quaternary ammonium compounds, have to be introduced into copperethanolamine preservative systems as well, to improve resistance of impregnated wood against copper tolerant fungi and insects.

Impregnated wood used outdoors (e.g. for decking, retaining walls, garden furniture, etc) is subjected to damaging effects of weathering, in addition to moisture and rainfall causing preservative leaching, especially to UV light. That is why majority of preservative-treated wood manufacturers recommend protection of impregnated wood with surface finishes (Feist and Ross 1995). However, interactions between impregnated wood and surface coatings are not always comparable to the ones between coatings and untreated wood. Literature search revealed that preservativetreated wood could be considered as an appropriate substrate for coatings (Kotama 1992; Bardage and Bjurman 1998). Several studies have demonstrated that application of surface finishes on impregnated wood can lead to enhanced surface stability and protection against weathering. However, in most of the studies, adhesion of finishes to CCA and CCB treated wood was studied (Feist and Ross 1995) and up to our best knowledge we were not able to find any data on properties of surface coatings on copper-amine treated wood. 


\section{Experimental}

\subsection{Material}

2.1a Specimens: Specimens were made of Norway spruce sapwood (Picea abies Karst.). The quality of the material met the requirements of the respective standards.

2.1b Treatment solutions: Two different treatment aqueous solutions, based on copper(II) sulphate and ethanolamine were used. Copper to ethanolamine molar ratio was set up to $(1: 6)$ in both solutions used. This ratio was chosen in order to achieve dissolution of all preservative ingredients. The first solution (CuE) contained copper(II) sulphate and ethanolamine only, whereas the second one (CuEQ) consisted of copper(II) sulphate, ethanolamine, octanoic acid and alkyl-diethyl-benzyl-ammonium-chloride. Concentration of alkyl-diethyl-benzyl-ammonium-chloride and $\mathrm{Cu}$ was $1.0 \%$. The detailed properties of preservative solutions are explained by Humar and coworkers (Humar et al 2005). For comparison, specimens impregnated with distilled water were prepared as well.

2.1c Coatings: In this study, two different commercially obtained wood finishes were used (table 1): opaque white pigmented medium build acrylic waterborne paint (OWW) and transparent non-pigmented medium build acrylic waterborne stain with UV filters and absorbers (TNW).

\subsection{Methods}

2.2a Impregnation of wood specimens: The specimens were vacuum impregnated according to the EN 113 procedure (EN 113 1989). Composition of preservatives influences solution uptakes of the wood specimens. Later, the specimens were conditioned for four weeks, the first two weeks in closed chambers, the third week in half closed and the fourth week in open ones; and afterwards stored at $25^{\circ} \mathrm{C}, 65 \% \mathrm{RH}$. 2.2b Leaching procedure: Leaching was performed according to the modified ENV 1250 procedure (ENV 1250 1994). The dimensions $(15 \times 25 \times 50 \mathrm{~mm})$ and orientations of the samples met the requirements of this standard. In total, 81 specimens were used. In order to speed up the experiment, the following two modifications of ENV 1250 (1994) were done: instead of five, three specimens were positioned in the same vessels and mixing of water was achieved by using a shaking device instead of a magnetic stirrer. For each combination of a preservative and a surface coating, nine specimens were prepared, that were leached in three separate vessels. All together, 27 vessels were prepared. The specimens were positioned in a vessel with a ballasting device. To this, $300 \mathrm{~g}$ of distilled water was added and the vessel with its content was exposed to shaking with a frequency of $55 \mathrm{~min}^{-1}$. Water was replaced seven times in ten leaching days. Leachates from the same vessels were collected and mixed together. Afterwards, atomic absorption spectroscopy (AAS) (Varian SpectrAA Duo FS240) analysis of the leachate was performed. Percentages of leached copper were calculated from the amount of retained copper determined gravimetrically and amount of copper in the collected leachate.

\section{2c Surface treatment and determination of surface coatings' characteristics:}

(i) Contact angle determination: For contact angle measurements, $70 \mathrm{~mm}$ wide radial surfaces from $(70 \times 20 \times 330 \mathrm{~mm})$ $(r \times t \times l)$ specimens were used. The samples were kept in a climate chamber at $20^{\circ} \mathrm{C}$ and $65 \%$ humidity, to obtain constant mass, determined by two sequential measurements within $24 \mathrm{~h}$. Static contact angles of water (W) or two different finishes (table 1) were measured as proposed by Scheikl and Dunky (1998). Contact angles $\theta$ were calculated with a presumption that a drop on a substrate is a part of a sphere (1) (Scheikl and Dunky 1998).

$$
\operatorname{tg} \frac{\theta}{2}=\frac{2 h}{D},
$$

Table 1. Some basic characteristics of applied coatings.

\begin{tabular}{lcccc}
\hline Code & $\begin{array}{c}\text { Resin/ } \\
\text { solvent }\end{array}$ & $\begin{array}{c}\text { Density } \\
\left(\mathrm{kg} / \mathrm{m}^{3}\right)\end{array}$ & $\begin{array}{c}\text { Surface tension } \\
\left(\mathrm{mJ} \cdot \mathrm{m}^{-2}\right)\end{array}$ & $\begin{array}{c}\text { Non-volatile- } \\
\text { matter content }\end{array}$ (\%) \\
\hline OWW & Acrylic (water) & 1160 & $33 \cdot 3$ & $42 \cdot 3$ \\
TNW & Acrylic (water) & 1060 & $35 \cdot 1$ & $34 \cdot 3$ \\
\hline
\end{tabular}

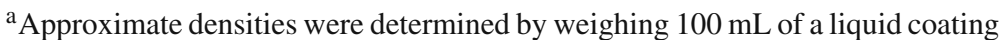

${ }^{\mathrm{b}}$ Surface tensions were measured by the Wilhelmy plate method with the Krüss K12 tensiometer, Krüss Gmbh Hamburg Germany, in the laboratories of LERMAB, Universite Henri Poincare, Faculte des Sciences, Nancy, France ${ }^{\mathrm{c}}$ Content of non-volatile matter was determined by the SIST EN ISO 3251 method (1997) 
where $\theta$ is a contact angle, $D$ a drop width and $h$ its height.

(ii) Penetration depth: The samples of $(30 \times 20 \times 50 \mathrm{~mm})$ $(r \times t \times l)$, free from defects, cracks or compression wood, were taken out of the samples of dimensions $(70 \times 20 \times$ $330 \mathrm{~mm})(r \times t \times l)$. Before penetration depth determination, the samples were conditioned at $20^{\circ} \mathrm{C}$ and $65 \%$ relative humidity. After conditioning, a cross-cut surface of a specimen was soaked into a coating (1 mm deep). Each coating was applied to cross-cut surfaces of three different substrates (CuE impregnated wood, CuEQ impregnated wood and non-impregnated wood; three parallel specimens per each substrate). The specimens were kept in contact with coatings for $5 \mathrm{~min}$. After soaking, the residual coatings on specimen surfaces were removed by a paper cloth. Immediately afterwards, the specimens were put into an oven $\left(103 \pm 2{ }^{\circ} \mathrm{C}, 1 \mathrm{~h}\right)$, followed by conditioning, as described earlier. Subsequently, small, oriented specimens were prepared $(20 \times 10 \times 10 \mathrm{~mm})$ $(r \times t \times l)$ with coatings on cross-cut sections. These small samples were then vacuum impregnated with distilled water (90\% vacuum) and again exposed to water for another hour at a pressure of 8 bar. Thin strips $(20 \mu \mathrm{m})$ were then microtomed from radial faces of blocks, using a conventional sliding microtome. The strips were stained for $1 \mathrm{~h}$ with Sudan IV. For each system, three samples were prepared. Penetration depth was determined (light microscope, magnification $=120 \times$ ) as the average of maximal penetration values on each sample.

(iii) Drying characteristics: To get some information on the drying characteristics of the coatings on different substrates, we carried out the standard test DIN 53150 (1971). After conditioning, for each test substrate a panel of dimensions $(70 \times 20 \times 330 \mathrm{~mm})(r \times t \times l)$ with inclination of the growth rings $(90 \pm 10)^{\circ}$ to the larger lateral test surface $(330 \times 70 \mathrm{~mm})$ was prepared and coated. Monitoring of the drying stage began immediately after coating application, as proposed in standard DIN 53150 (1971).

(iv) Adhesion-pull-off test: Adhesion of coatings to the test substrates was determined by the modified standard pulloff test EN 24624 (1997). One conditioned panel of each test substrate (CuE impregnated wood, CuEQ impregnated wood and non-impregnated wood $)$ of dimensions $(70 \times 20 \times$ $400 \mathrm{~mm})(r \times t \times l)$ with inclination of the growth rings $(90 \pm$ $10)^{\circ}$ to the larger lateral test surface were finished with three layers in one of the test finishes, with $24 \mathrm{~h}$ drying periods between the first, the second and the third application. After three weeks of drying of a surface coating system, the panels were prepared for gluing of test cylinders on the coated surface. A two-component, epoxy-based adhesive was evenly applied to a freshly-cleaned surface of a test cylinder (a steel faced cylinder having a diameter of $11.25 \mathrm{~mm}$, instead of $20 \mathrm{~mm}$, as determined in the EN 24624 (1997) standard. The adhesive-coated face of the test cylinder was placed in contact with a coating for $24 \mathrm{~h}$. After $24 \mathrm{~h}$, the cutting device was used to cut around the circumference of the test cylinder through to the substrate. On each panel, ten cylinders were applied. Afterwards, larger panels were cut to smaller specimens which were then placed into the tensile tester (pulloff adhesion tester by DeFelsko). Tensile stresses to break the test assemblies were recorded and fracture surfaces examined. When there was less than $40 \%$ of a test cylinder area covered by a coating, an adhesive type failure (A) of the surface system was presumed. Otherwise the failure type was regarded as a cohesive one $(\mathrm{K})$.

(v) Adhesion-cross-cut test: Adhesion was in addition to a pull-off test, assessed also by a cross-cut test EN ISO 2409 (1997). Researchers cut coating approximately $45^{\circ}$ to the direction of the grain with the cutting tool, which have a spacing of $1 \mathrm{~mm}$ or $2 \mathrm{~mm}$. Second cut was crossing the original cuts at $90^{\circ}$, so that a lattice pattern was formed. We brushed the panel lightly and carefully examined the cut area with the six-step classification, which is given in standard EN ISO 2409 (1997). In addition, the dry film coating thickness was determined (microscopic method, EN ISO 2808 (1997)).

\section{Results and discussion}

\subsection{Retention}

Retention of the preservatives in the specimens was very high. On an average, specimens retained $30 \mathrm{~kg} / \mathrm{m}^{3}$ of $\mathrm{CuE}$ and $44 \mathrm{~kg} / \mathrm{m}^{3}$ of CuEQ. Recommended retention levels for wood used in class 4 (ground contact or fresh water contact) are usually lower (Willeitner 2001), but it has to be taken into account that in our preservative solutions there were significant amounts of ethanolamine and octanoic acid. These additives were introduced into preservative solutions in order to improve copper fixation and not just for their fungicidal effect. Furthermore, the authors chose higher than recommended retention levels in order to maximize influence of impregnated wood on surface coatings.

Anyway, two contradictory recommendations on necessary preservative loadings in wood can be found in the literature. Willeitner (2001) reported that retention of $5 \mathrm{~kg} / \mathrm{m}^{3}$ of copper-ethanolamine based Wolmanit CX-8 is enough to protect non-durable wood in ground contact. On the contrary, Nordic Wood Preservation Council recommends notably higher retention levels. For example, retention of $36 \mathrm{~kg} / \mathrm{m}^{3}$ of copper-amine-quats was suggested for wood to be used in class 4 (Hughes 2004). So, we assume that retentions achieved in our experiment meet the requirements of Nordic Wood Preservation Council.

\subsection{Contact angles of coating on preserved wood}

As can be seen from table 2, water exhibited the best wetting of CuEQ impregnated wood $\left(\theta<10^{\circ}\right)$, followed by wetting of non-preserved wood $\left(42.7^{\circ}\right)$ and finally by contact angles on CuE-treated wood. Higher contact angles of water implies more hydrophobic surfaces and is well known 
(Gerardin et al 2007). They reflect their higher surface free energies. Generally, the same pattern of contact angles on various substrates was observed also with the waterborne coatings: the lowest contact angles were observed on CuEQ impregnated wood $\left(\mathrm{OWW}, 63 \cdot 1^{\circ}\right.$; TNW, 57.0 $)$, while the highest ones on $\mathrm{CuE}$ treated wood (OWW, 88.4\% $\left.78 \cdot 4^{\circ}\right)$.

\subsection{Penetration depth}

Both coatings exhibited the deepest penetration into wood, impregnated with CuEQ (OWW $1190 \mu \mathrm{m}$, TNW $2660 \mu \mathrm{m}$; table 3). At CuE impregnated wood and at non-treated

Table 2. Average contact angles $(\bar{X})$ of opaque white pigmented medium build acrylic waterborne paint (OWW), transparent non-pigmented medium build acrylic waterborne stain with UV filters and absorbers (TNW) and water on different substrates.

\begin{tabular}{|c|c|c|c|c|c|c|}
\hline \multirow{3}{*}{ Pretreatment } & \multicolumn{6}{|c|}{ Contact angle $\left({ }^{\circ}\right)$} \\
\hline & \multicolumn{2}{|c|}{ OWW } & \multicolumn{2}{|c|}{ TNW } & \multicolumn{2}{|c|}{ Water } \\
\hline & $\bar{X}$ & $\sigma$ & $\bar{X}$ & $\sigma$ & $\bar{X}$ & $\sigma$ \\
\hline $\mathrm{CuE}$ & $88 \cdot 4$ & $5 \cdot 0$ & $78 \cdot 4$ & $8 \cdot 1$ & 59.6 & $9 \cdot 8$ \\
\hline CuEQ & $63 \cdot 1$ & $6 \cdot 5$ & $57 \cdot 0$ & $3 \cdot 6$ & $<10 \cdot 0$ & - \\
\hline None & $87 \cdot 1$ & $5 \cdot 7$ & 67.5 & $4 \cdot 0$ & 42.7 & $8 \cdot 6$ \\
\hline
\end{tabular}

Table 3. Penetration of opaque white pigmented medium build acrylic waterborne paint (OWW) and transparent non-pigmented medium build acrylic waterborne stain with UV filters and absorbers (TNW) coatings into various substrates.

\begin{tabular}{lcc}
\hline & \multicolumn{2}{c}{ Penetration depth $(\mu \mathrm{m})$} \\
\cline { 2 - 3 } & OWW & TNW \\
Pretreatment & $\bar{X}$ & $\bar{X}$ \\
\hline CuE & 371 & $/$ \\
CuEQ & 1190 & 2660 \\
None & 350 & 980 \\
\hline
\end{tabular}

wood, the authors observed approximately the same and substantially lower penetration depth of the opaque pigmented waterborne finish OWW (371 $\mu \mathrm{m}$ and $350 \mu \mathrm{m}$, respectively). This observation is in agreement with the contact angle data (table 2): we measured higher penetration depth into CuEQ treated wood, where compared to the $\mathrm{CuE}$ preserved and non-treated substrates lower contact angles were observed. The penetration depth of the transparent non-pigmented coating TNW into non-impregnated wood was $980 \mu \mathrm{m}$. However, the authors did not succeed in measuring the penetration depth of TNW into CuE impregnated wood, while during preparation and microtomization, the coating was pulled out from the substrate.

De Meijer and coworkers (De Meijer et al 1998) report that penetration depth is influenced by the free surface energy of the substrate. Substrate with a higher surface free energy is more easily wetted by a liquid, and its penetration depth is higher. We believe that these statements are reflected also in our results (contact angles in table 2 and penetration depths in table 3), although our research team did not make any attempts to measure and explain surface free energies of $\mathrm{CuE}$ and CuEQ preserved substrates.

\subsection{Drying characteristics}

Drying times for certain drying stages are presented in table 4. The results show that pretreatment of wood with preservatives has a pronounced influence on time, necessary to reach certain drying stages, especially the last stage 7 . The fastest drying of both finishes (drying stage 7) was observed on non-impregnated wood, while the coatings on impregnated wood dried substantially slowly. The same observation was reported by Pavlič and coworkers (Pavlič et al 2005) for coatings on boron-based preservative impregnated specimens. In addition, it was also noticed that both finishes require different drying periods. On all three substrate types, the drying stage 7 was achieved first by the transparent coating, and later by the pigmented finish OWW. Faster drying of the finish TNW in comparison with drying of OWW can be attributed to deeper penetration of the transparent TNW stain. The absorption rate of the liquid phase of the formulation TNW into wood is larger, resulting in faster film formation on the surface.

Table 4. Drying time values of first coat for specific drying stages.

\begin{tabular}{|c|c|c|c|c|c|c|}
\hline \multicolumn{2}{|l|}{ System } & \multirow[b]{2}{*}{ Coating spreading rate $\left(\mathrm{g} / \mathrm{m}^{2}\right)$} & \multicolumn{4}{|c|}{ Drying time for selected drying stage (min) } \\
\hline Substrate pretreatment & Coating & & $2(20 \mathrm{~g})$ & $3(200 \mathrm{~g})$ & $5(2 \mathrm{~kg})$ & $7(20 \mathrm{~kg})$ \\
\hline $\mathrm{CuE}$ & OWW & 79,6 & $13 \min$ & $22 \min$ & $41 \mathrm{~min}$ & $2 \mathrm{~h} 04 \mathrm{~min}$ \\
\hline CuEQ & OWW & 79,6 & $15 \min$ & $29 \mathrm{~min}$ & $43 \mathrm{~min}$ & $2 \mathrm{~h} 50 \mathrm{~min}$ \\
\hline None & OWW & 79,6 & $12 \min$ & $19 \mathrm{~min}$ & $32 \mathrm{~min}$ & $1 \mathrm{~h} 17 \mathrm{~min}$ \\
\hline $\mathrm{CuE}$ & TNW & 99,8 & $06 \min$ & $12 \mathrm{~min}$ & $21 \mathrm{~min}$ & $1 \mathrm{~h} 31 \mathrm{~min}$ \\
\hline CuEQ & TNW & 99,8 & $07 \mathrm{~min}$ & $13 \mathrm{~min}$ & $18 \mathrm{~min}$ & $1 \mathrm{~h} 19 \mathrm{~min}$ \\
\hline None & TNW & 99,8 & $11 \mathrm{~min}$ & $18 \mathrm{~min}$ & $24 \mathrm{~min}$ & $53 \mathrm{~min}$ \\
\hline
\end{tabular}




\subsection{Adhesion, determined by pull-off test}

Average thicknesses of the coating systems, to be tested for their adhesion, are presented in table 5. Coating thickness of TNW was higher on all substrates than coating thickness of OWW. Coating thickness depends on non-volatile-matter content, wet coating thickness and also on penetration depth. However, we did not find any relationship between those parameters, and were not able to identify the key factors that influence those parameters.

The adhesion values of the coatings varied between 3.8 $\mathrm{MPa}$ and 4.7 MPa, depending on the coating and substrate types (table 6). The strongest adhesion value of the coating OWW (opaque pigmented acrylic waterborne finish) was exhibited on CuEQ impregnated wood (4.4 MPa), and it was somewhat lower both on $\mathrm{CuE}$ impregnated wood (3.9 MPa) and on non-impregnated wood (3.8 MPa). TNW exhibited approximately the same adhesion on CuEQ impregnated as well as on non-treated wood (4.6-4.7 MPa). A bit lower value of this coating was recorded on $\mathrm{CuE}$ impregnated wood $(4 \cdot 2 \mathrm{MPa})$. It has to be noted that the value for OWW on non-impregnated wood is not realistic since the failure was predominantly of a cohesive type. Nevertheless, this means that the true adhesion value was greater than $3.8 \mathrm{MPa}$, as measured for the mentioned system. Generally, all the adhesion values met the required values since they were larger than $2 \mathrm{MPa}$, which is considered to be the minimal acceptable value (Oblak and Kričej 2005). The authors

Table 5. Thicknesses of coatings OWW (opaque pigmented acrylic waterborne finish) and TNW (transparent acrylic waterborne finish) on various substrates.

\begin{tabular}{lcc}
\hline & \multicolumn{2}{c}{ Thickness $(\mu \mathrm{m})$} \\
\cline { 2 - 3 } Substrate pretreatment & OWW & TNW \\
\cline { 2 - 3 } & $\bar{X}$ & $\bar{X}$ \\
\hline CuE & 36,0 & 47,0 \\
CuEQ & 41,5 & 44,2 \\
None & 30,4 & 47,0 \\
\hline
\end{tabular}

can conclude that our results fit well into more or less general scheme: pretreatment of wood with various preservatives generally do not decrease adhesion of finishes; in some cases even an increase of adhesion of finishes on pretreated wood has been reported (Feist and Ross 1995; Bardage and Bjurman 1998; Pavlič et al 2005).

\subsection{Adhesion, determined by cross-cut test}

According to the standard EN ISO 2409 (1997) method, acceptable adhesion is reached, when assessed from 0 to 2 . In our case (spacing of the cuts $1 \mathrm{~mm}$ ), three above-limit values were found: OWW on CuEQ impregnated wood (Humar et al 2005) and TNW on CuE impregnated wood (3) and on non-impregnated wood (3) (table 7: Adhesion of the coatings OWW and TNW (cross-cut test)). However, the test with the knife with $1 \mathrm{~mm}$ spacing is intended for measurements of coatings which are up to $60 \mu \mathrm{m}$ thick and applied on hardwoods. As authors used softwood (spruce), assessment of adhesion, obtained by a $2 \mathrm{~mm}$ spacing knife was in compliance with the requirements of the standard EN ISO 2409 (1997).

\subsection{Leaching}

From the specimens, impregnated with a preservative solution containing copper and ethanolamine $(\mathrm{CuE}), 7.6 \%$ of

Table 7. Adhesion of coatings OWW and TNW (cross-cut test) on various pretreated specimens.

\begin{tabular}{|c|c|c|c|c|}
\hline \multirow{3}{*}{ Pretreatment } & \multicolumn{4}{|c|}{ Assessment of adhesion } \\
\hline & OWW & TNW & OWW & TNW \\
\hline & \multicolumn{2}{|c|}{ Knife $1 \mathrm{~mm}$} & \multicolumn{2}{|c|}{ Knife $2 \mathrm{~mm}$} \\
\hline $\mathrm{CuE}$ & 2 & 3 & 2 & 2 \\
\hline CuEQ & 3 & 1 & 2 & 1 \\
\hline None & 2 & 3 & 2 & 2 \\
\hline
\end{tabular}

Table 6. Adhesion values (pull-off test) and predominant types of failure.

\begin{tabular}{|c|c|c|c|c|c|c|c|c|}
\hline \multirow[b]{4}{*}{ Treatment } & \multicolumn{8}{|c|}{ Adhesion (MPa) and failure type (adhesive (A) or cohesive $(\mathrm{K})$ in \%) } \\
\hline & \multicolumn{4}{|c|}{ OWW } & \multicolumn{4}{|c|}{ TNW } \\
\hline & \multicolumn{2}{|c|}{ Adhesion } & \multicolumn{2}{|c|}{ Failure type } & \multicolumn{2}{|c|}{ Adhesion } & \multicolumn{2}{|c|}{ Failure type } \\
\hline & $\bar{X}$ & $\sigma$ & $\bar{X}$ & $\sigma$ & $\bar{X}$ & $\sigma$ & $\bar{X}$ & $\sigma$ \\
\hline $\mathrm{CuE}$ & 3,9 & 0,3 & $29(\mathrm{~A})$ & 17 & 4,2 & 0,8 & $28(\mathrm{~A})$ & 15 \\
\hline CuEQ & 4,4 & 0,5 & $36(\mathrm{~A})$ & 8 & 4,6 & 0,5 & $20(\mathrm{~A})$ & 10 \\
\hline None & 3,8 & 0,3 & $62(\mathrm{~K})$ & 19 & 4,7 & 0,5 & $26(\mathrm{~A})$ & 8 \\
\hline
\end{tabular}


Table 8. Copper leaching from the Norway spruce specimens treated with two different copper-ethanolamine based solutions. Part of the specimens was prior to leaching surface coated with two different finishes. Standard deviations are given in the parentheses.

\begin{tabular}{llc}
\hline Pretreatment & Applied coating & Leached copper (\%) \\
\hline \multirow{2}{*}{$\mathrm{CuE}$} & None & $7 \cdot 6(1 \cdot 0)$ \\
& Acrylic waterborne stain, white, non-transparent (OWW) & $0 \cdot 2(0 \cdot 1)$ \\
& Acrylic waterborne stain, transparent with UV absorber (TNW) & $0 \cdot 3(0 \cdot 0)$ \\
$\mathrm{CuEQ}$ & None & $6 \cdot 7(0 \cdot 5)$ \\
& Acrylic waterborne stain, white, non-transparent (OWW) & $3 \cdot 3(0 \cdot 4)$ \\
& Acrylic waterborne stain, transparent with UV absorber (TNW) & $1 \cdot 2(0 \cdot 2)$ \\
\hline
\end{tabular}

$\mathrm{Cu}$ was leached on an average (table 8). Addition of other co-biocides and octanoic acid into the preservative solution resulted in slightly reduced copper leaching: approximately $10 \%$ lower leaching rates were determined at specimens, impregnated with CuEQ (6.7\%). We presume that the main reasons for the observed difference originate in hydrophobic effect of octanoic acid and new less soluble complexes between copper, ethanolamine, quaternary ammonium compound and octanoic acid, formed in the treated wood (Humar et al 2007). However, it has to be considered that retentions of preservative solutions were relatively high. Lower retentions generally result in lower leaching rates (Humar et al 2007).

Surface coatings significantly decreased leaching of copper from the impregnated wood. At specimens, impregnated with $\mathrm{CuE}$ and surface treated with two different acrylic waterborne finishes, comparable leaching rates were determined, on an average between $0.2 \%$ and $0.3 \%$ (table 8 ). These leaching rates are relatively low and they are comparable to leaching from uncoated copper-chromium-arsenic (CCA) treated specimens (Temiz et al 2006). On the other hand, notably higher leaching rates were determined from CuEQ impregnated and surface coated specimens. From CuEQ impregnated specimens, surface treated with the acrylic waterborne non-transparent stain, on an average 3.3\% of $\mathrm{Cu}$ was leached, which is 10 times more than from $\mathrm{CuE}$ impregnated specimens, coated with the same acrylic finish. Slightly better results were determined at CuEQ treated wood, surface coated with the acrylic transparent waterborne stain, containing UV absorbers (TNW). The authors could not connect the data on leaching of $\mathrm{Cu}$ from the preserved and coated wood with characteristics of surface coating systems. The following speculation could be made from drying times: longer drying times of the coatings on CuEQ impregnated wood, compared to the drying times on $\mathrm{CuE}$ treated wood, could indicate some interferences of the CuEQ constituents (octanoic acid and/or quats) with the coatings drying mechanisms and film formation processes, resulting in their different structures and influencing on leaching of the preservative through the coating layer. However, this is just a speculation requiring extensive experimental work to be proven or not. It might also be remembered that the water repellent effect of octanoic acid that has a positive influence on performance of copper-ethanolamine impregnated wood in service negatively influences performance of acrylic stains with spruce wood, impregnated with CuEQ.

\section{Conclusions}

Impregnation of spruce wood with copper-ethanolamine based wood preservatives has an influence on certain characteristics of waterborne acrylic coatings applied to the wooden substrates. In the case of wood, impregnated with CuEQ (containing copper(II) sulphate, ethanolamine, boron compound and quaternary ammonium compound), better wetting by surface waterborne acrylic coatings and their higher penetration depths were observed. Improved adhesion of coatings on CuEQ impregnated wood compared to adhesion on $\mathrm{CuE}$ treated wood was exhibited as well. In general, the authors conclude that characteristics of surface finishes on copper-ethanolamine based preservative treated wood were just adequate as those on untreated spruce wood substrates.

It was also shown that copper leaching was significantly reduced by application of waterborne acrylic surface finishes on preservative treated wood. Addition of other co-biocides (boron compound, quaternary ammonium compound, octanoic acid) to copper and ethanolamine containing preservative formulations revealed a negative impact on prevention of leaching through the applied coating layers. Time period required to obtain completely dry films on wood impregnated with $\mathrm{CuEQ}$ were longer, compared to drying times of finishes on $\mathrm{CuE}$ and especially on non-treated wood. Consequently, the resulting film structure might be different on CuEQ impregnated wood, causing lower protective efficacy against leaching, compared to efficacy against leaching through films on $\mathrm{CuE}$ pretreated wood. However, this presumption should be the matter of further research work.

\section{Acknowledgement}

The authors would like to acknowledge the Slovenian Research Agency for financial support in the frame of the programs L4-6209-0481 and L4-7163-0481. 


\section{References}

Bardage S L and Bjurman J 1998 J. Coat. Technol. 7939

Cao J and Kamdem D P 2004 Holzforschung $\mathbf{5 8} 32$

De Meijer M, Thurich K and Militz H 1998 Wood Sci. Technol. 32 347

DIN 531501971 Prüfung von Anstrichstoffen und änlichen Beschictungsstoffen - Bestimmung des Trockengrades von Abstrichen (Abgewandeltes Bandow-Wolf-Verfahren)

EN 1131989 Wood preservatives; determination of the toxic values against wood destroying basidiomycetes cultured in agar medium

EN 246241997 Paints and varnishes-Pull-off test (ISO 4624:1978)

EN ISO 24091997 Paints and varnishes-Cross-cut test (ISO 2409:1992)

EN ISO 28081997 Paints and varnishes-Determination of film thickness (ISO 2808)

EN ISO 32511997 Paints, varnishes and plastics - Determination of non-volatile-matter content

ENV 12501994 Wood preservatives-Methods for measuring losses of active ingredients and other preservative ingredients from treated timber-Part 2: Laboratory method for obtaining samples for analysis to measure losses by leaching into water or synthetic sea water

Feist W C and Ross A S 1995 For. Prod. J. 4529

Gerardin P, Petrič M, Petrissans M and Lambert J J 2007 Polym. Degrad. Stab. 92653

Hughes A S 2004 The tools at our disposal (Brussels: COST E22 environmental optimization of wood protection)

Humar M, Žlindra D and Pohleven F 2007 Build. Environ. 42578

Humar M, Kalan P, Šentjurc M and Pohleven F 2005 Wood Sci. Technol. 39685

Kotama L 1992 The International Research Group on Wood Preservation, Document No. IRG/WP 3684-92

Oblak L and Kričej B 2005 Zb. Gozd. Lesar. 76147

Pavlič M, Kričej B, Tomažič M and Petrič M 2005 Surf. Coat. Int. Part B Coat. Trans. 8841

Scheikl M and Dunky M 1998 Holzforschung 5289

Temiz A, Yildiz U C and Nilsson T 2006 Build. Environ. 41910

Ung Y T and Cooper P A 2005 Holz Roh- Werkst. 63186

Willeitner H 2001 Current national approaches to defining retentions in use (Brussels: COST E22 environmental optimization of wood protection) 